\title{
Assisted hopping and interaction effects in impurity models.
}

\author{
L. Borda ${ }^{1}$ and F. Guinea ${ }^{2}$ \\ 1 Department für Physik and CENS, \\ Ludwig-Maiximilians-Universität München Theresienstr. 37, \\ D-80333 Munich, Germany \\ ${ }^{2}$ Instituto de Ciencia de Materiales de Madrid, \\ CSIC, Cantoblanco, E-28049 Madrid, Spain
}

(Dated: November 20, 2018)

\begin{abstract}
We study, using Numerical Renormalization Group methods, the generalization of the Anderson impurity model where the hopping depends on the filling of the impurity. We show that the model, for sufficiently large values of the assisted hopping term, shows a regime where local pairing correlations are enhanced. These correlations involve pairs fluctuating between on site and nearest neighbor positions.

PACS numbers: 75.30.Mb, 73.22.Gk, 73.23.Hk, 05.10.Cc
\end{abstract}

\section{INTRODUCTION.}

The formation of local moments is the simplest and most extensively studied manifestation of strong electron-electron repulsion in impurities in metals 1.2 .3 . This phenomenon can also be observed in mesoscopic systems coupled to metallic leads..5. In the opposite limit, when the "impurity" becomes a metallic grain whose electronic structure needs to be described by many narrowly spaced levels, the electron-electron repulsion leads to Coulomb blockade $\boldsymbol{\sigma}^{\underline{\underline{6}}}$. The intermediate regime when the energy scales of interest, the temperature, the strength of the Coulomb repulsion and the level spacing are not too different in magnitude is not so well understood. A similar situation arises in the study of strongly correlated systems. The analogous of the Anderson impurity model is the Hubbard model ${ }^{7.8}$ for extended systems, which leads to a Mott transition and the formation of local moments at half filling. The opposite limit, when many levels within the unit cell have to be included, leads, generically, to ordinary Fermi liquid behavior.

The simplest modifications of the extended Hubbard model taking into account the multiplicity of levels in the ions in the unit cell lead to assisted hopping terms ${ }^{9,10}$, which modify substantially the phase diagram, and tend to favor superconductivity. Analogously, if the internal degrees of freedom of the metallic grain are included, beyond the constant interaction term which leads to Coulomb blockade, one finds non equilibrium effects 11.12 which suppress the Coulomb blockade. These effects are similar to the formation of an excitonic resonance found in the excitation of core electrons ${ }^{13}$. One can also extend the Anderson impurity model to include the effects associated to the existence of many orbitals. When the influence of these orbitals is described in terms of effective interactions within the restricted Hilbert space of the usual Anderson model, one indeed finds assisted hopping terms 14 . The resulting model has been analyzed using a mean field, BCS like decoupling of the interaction term 14 , and by the flow equation method ${ }^{15}$ (for a description of the method, see ${ }^{16.17}$ ), which is well suited to the analysis of impurity models 18.19 .20 .21 .22 . These works show a tendency towards local pairing away from half filling, in agreement with the studies of bulk systems ${ }^{9.10}$. We present here more accurate calculations, using the $\mathrm{Nu}$ merical Renormalization Group Method ${ }^{23}$, which characterize the low energy properties of the model in a numerically exact way.

We examine the effect of the assisted hopping term on the local density of states and find that with increasing assisted hopping amplitude the peak characteristic to the mixed valence regime of the Anderson model gets broadened and shifted to negative frequencies. This result is consistent with the fact that the assisted term enhances the occupation of the local level, also found in the calculations. To demonstrate the effect of the new term on the formation of the local moment, we compute the local spin spectral function and find that the spin susceptibility gets suppressed as the assisted hopping term is turned on. This suppression might be interpreted as the trace of the pairing. We also check the pairing correlations in a more direct way and calculate the related spectral functions. Our main result is that -although the effect of the assisted term on the pairing correlations on the local level could be explained qualitatively by just considering the renormalization of the level- the so-called off diagonal correlations (pairing between the $d$-electron and the conduction electrons located at the impurity position) are also enhanced.

The paper is organized as follows: The model, and the method of calculation are described in the next section. Then, the main results are presented. Section IV gives the main conclusions of our work. 


\section{THE MODEL AND METHOD OF CALCULATION.}

\section{A. The model}

We describe the effects of the internal degrees of freedom of the impurity in terms of effective interactions defined within the restricted Hilbert space of the standard Anderson impurity 14 . Using perturbation theory, this approach is justified when the typical level spacing within the impurity, $\Delta$, is much smaller than value of the Coulomb interaction, $U$. Then, it is easy to show that the Anderson model is recovered in the limit $\Delta / U \rightarrow \infty$. We will study the Hamiltonian:

$$
\begin{aligned}
\mathcal{H} & =\mathcal{H}_{K}+\mathcal{H}_{i m p}+\mathcal{H}_{h y b}+\mathcal{H}_{\text {assisted }} \\
\mathcal{H}_{K} & =\sum_{k, s} \epsilon_{k} c_{k, s}^{\dagger} c_{k, s} \\
\mathcal{H}_{i m p} & =\epsilon_{d} n_{d}+U n_{d,+} n_{d,-} \\
\mathcal{H}_{h y b} & =\sum_{k, s} V\left(c_{k, s}^{\dagger} d_{s}+d_{s}^{\dagger} c_{k, s}\right) \\
\mathcal{H}_{\text {assisted }} & =\sum_{k, s} d V n_{d, s}\left(c_{k,-s}^{\dagger} d_{-s}+d_{-s}^{\dagger} c_{k,-s}\right)
\end{aligned}
$$

where $\mathcal{H}_{A}=\mathcal{H}_{K}+\mathcal{H}_{i m p}+\mathcal{H}_{h y b}$ is the Anderson Hamiltonian, and the assisted hopping terms are included in $\mathcal{H}_{\text {assisted }}$. We have also defined $n_{d, s}=d_{s}^{\dagger} d_{s}$ and $n_{d}=n_{d,+}+n_{d,-}$. The parameter $d V$ determines the strength of the assisted hopping effects. For the sake of simplicity we consider a flat band with constant density of states for the conduction electrons, with half bandwidth $D$. In the following we use $D$ as the energy unit.

\section{B. The Numerical Renormalization Group Method.}

To compute different quantities numerically we use Wilson's Numerical Renormalization Group method 23.24. In this method -after the logarithmic discretization of the conduction band - one maps the original impurity Hamiltonian onto a semi-infinite chain with the impurity at the end. One can show that as a consequence of the logarithmic discretization the hopping along the chain decreases exponentially, $t_{n} \sim \Lambda^{-n / 2}$ where $\Lambda$ is the discretization parameter, and $n$ is the index of the site in the chain. The separation of the energy scales due to the decreasing hopping provides the possibility to diagonalize the chain Hamiltonian iteratively and keep the states with the lowest lying energy eigenvalues as most relevant ones. Since we know the energy eigenstates and eigenvalues, we can calculate thermodynamical and dynamical quantities directly (e.g. spectral functions using their Lehman representation).

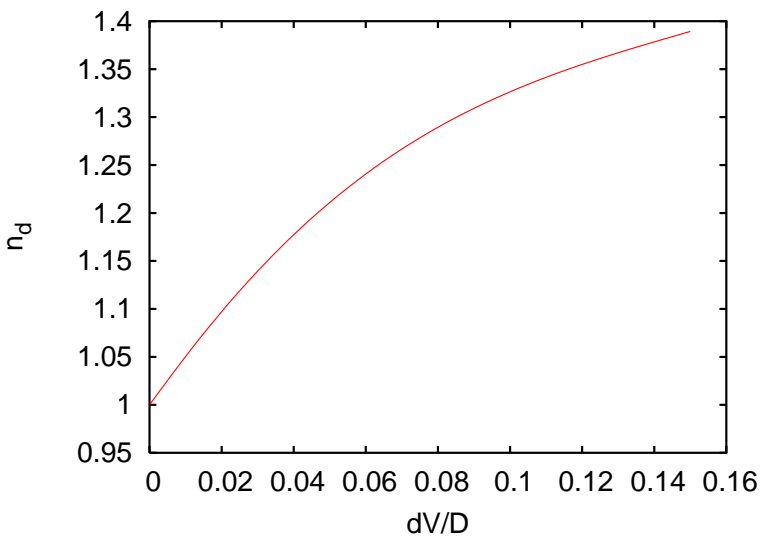

FIG. 1: Value of $n_{d}$ as function of $d V$ for $V=0.2, \epsilon_{d}=0$ and $U=0.05$.

\section{RESULTS}

The model has four parameters, $\epsilon_{d}, U, V$ and $d V$. Previous studies 14,15 suggest that the most significant deviations from the standard Anderson impurity model take place when the filling of the $d$ orbital is such that $1.2 \leq\left\langle n_{d}\right\rangle \leq 1.8$. We focus our attention to that regime as well.The filling is determined by $\epsilon_{d}, U$ and also by $d V$. The mean field decoupling of the assisted hopping term shows that the latter term contributes to the renormalizationof the position of the level. The value of $n_{d}$ as function of $d V$ is given in Fig. 11. Since the assisted hopping term renormalizes the impurity level downwards, that term favors the occupancy of the impurity level.

The fact that the assisted hopping term, $d V$, and the electron-electron repulsion $U$ have opposite effects can be appreciated in Fig. 2] where the influence of the value of $U$ on $n_{d}$, for a fixed value of $d V$, is shown.

Fig. [3] shows the imaginary part of theone particle Green's function associated to the localized level, defined as:

$$
\begin{aligned}
\frac{1}{\pi} G_{d}(\omega) & =\sum_{m, \sigma}\left|\left\langle 0\left|d_{\sigma}^{\dagger}\right| m\right\rangle\right|^{2} \delta\left(\omega-\epsilon_{m}\right) \\
& +\sum_{m, \sigma}\left|\left\langle 0\left|d_{\sigma}\right| m\right\rangle\right|^{2} \delta\left(\omega+\epsilon_{m}\right),
\end{aligned}
$$

where $|0\rangle(|m\rangle)$ is the ground ( $m$ th excited) state of the full Hamiltonian with energy $\epsilon_{0}=0\left(\epsilon_{m}\right)$.

In presence of no assisted hopping, the local density of states exhibits a broad peak at the Fermi level which is characteristic to the mixed valence regime of the Anderson impurity model. As $d V$ is increased from zero, the results show a broadening of the resonance, also consistent with the fact that, at the mean field level, the assisted hopping term modifies the effective one particle hopping. As one can see in Fig[3], the resonance gets not 


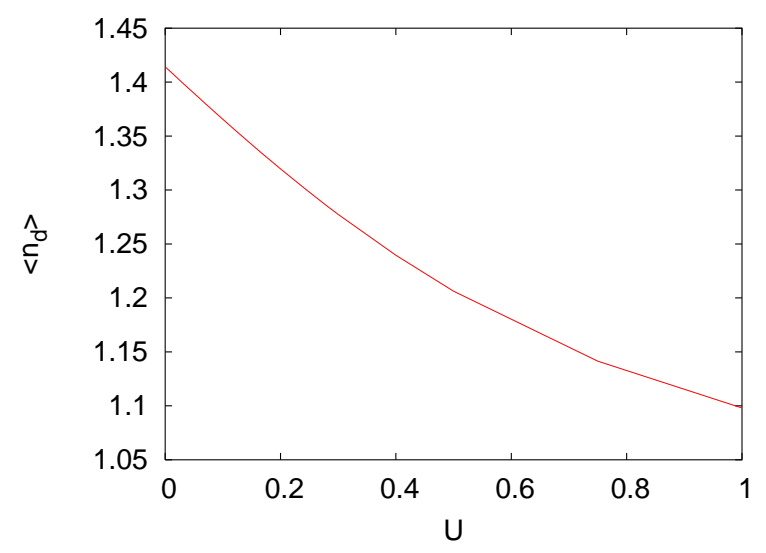

FIG. 2: Value of $n_{d}$ as function of $U$ for $V=0.2, \epsilon_{d}=0$ and $d V=0.15$.

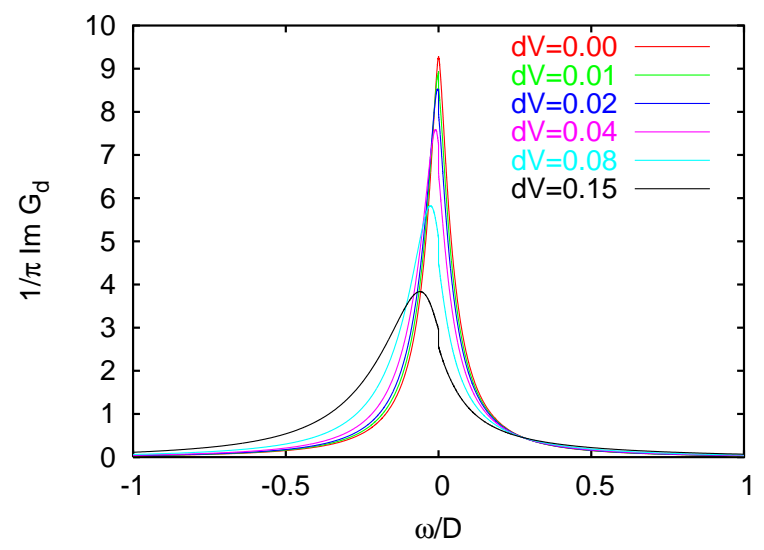

FIG. 3: Imaginary part of the impurity one electron Green's function, $1 / \pi G_{d}(\omega)$, for $V=0.2, \epsilon_{d}=0$ and $U=0.05$.

only broadened but also shifted down to negative energies. This fact could already be foreseen from the result for the occupation, since the occupation (at $T=0$ ) is just the integral of the density of states for negative frequencies. This enhancement of the occupation must leave its trace on the local moment formation as well.

The influence of the assisted hopping term on the for-

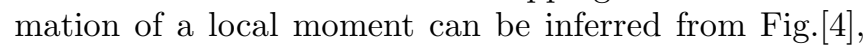

which displays the spin-spin correlation function:

$$
\operatorname{Im} \varrho_{S}^{z}(\omega)=\frac{1}{2} \sum_{n}\left|\left\langle 0\left|n_{d \uparrow}-n_{d \downarrow}\right| n\right\rangle\right|^{2} \delta\left(\omega-\epsilon_{n}\right)
$$

This function, for $d V=0$ shows a pronounced peak at low energies, which tend to the Kondo temperature below which the local moment is quenched as $U$ increases. At higher energies, $\operatorname{Im} \varrho_{S}^{z}(\omega) \sim \omega^{-1}$, which corresponds to a Curie susceptibility as function of temperature. This

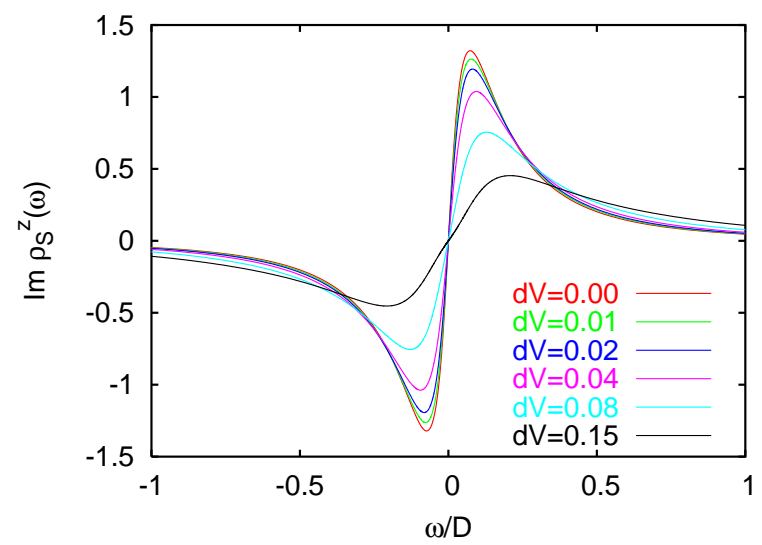

FIG. 4: Spin-spin correlation function for $V=0.2, \epsilon_{d}=0$ and $U=0.05$ and different values of $d V$.

behavior crosses over to a $\operatorname{Im} \varrho_{S}^{z}(\omega) \sim \omega$ regime which is characteristic to the compensation of the moment. (Alternatively, one could say that for very high frequencies $\operatorname{Im} \varrho_{S}^{z}(\omega) \sim \omega^{-1}$ as a consequence of the constant time correlator of the spin for very short times, while for small frequencies $\operatorname{Im} \varrho_{S}^{z}(\omega)$ shows a linear dependence on $\omega$ corresponding to the $\sim t^{-2}$ asymptotics of the correlation. As $d V$ is turned on, this peak becomes broader and it is shifted towards higher energies indicating the suppression of the local moment. This suppression is also consistent with the previous results and might be considered as a fingerprint of the local pairing.

To get a more direct insight to the relation of the assisted hopping and the pairing, we now analyze the possible existence of pairing correlations in the model. Within the reduced Hilbert space of the Anderson's impurity model, we can define two types of such correlations:

$$
\begin{aligned}
F(\omega) & =\sum_{n}\left|\left\langle 0\left|d_{\uparrow}^{\dagger} d_{\downarrow}^{\dagger}\right| n\right\rangle\right|^{2} \delta\left(\omega-\epsilon_{n}\right)+\left|\left\langle 0\left|d_{\uparrow} d_{\downarrow}\right| n\right\rangle\right|^{2} \delta\left(\omega+\epsilon_{n}\right) \\
F^{\prime}(\omega) & =\sum_{n}\left\langle 0\left|d_{\uparrow}^{\dagger} d_{\downarrow}^{\dagger}\right| n\right\rangle\left\langle n\left|\left(d_{\uparrow} c_{\downarrow}-d_{\downarrow} c_{\uparrow}\right)\right| 0\right\rangle \delta\left(\omega-\epsilon_{n}\right)+\left\langle 0\left|d_{\uparrow} d_{\downarrow}\right| n\right\rangle\left\langle n\left|\left(d_{\uparrow}^{\dagger} c_{\downarrow}^{\dagger}-d_{\downarrow}^{\dagger} c_{\uparrow}^{\dagger}\right)\right| 0\right\rangle \delta\left(\omega+\epsilon_{n}\right)
\end{aligned}
$$




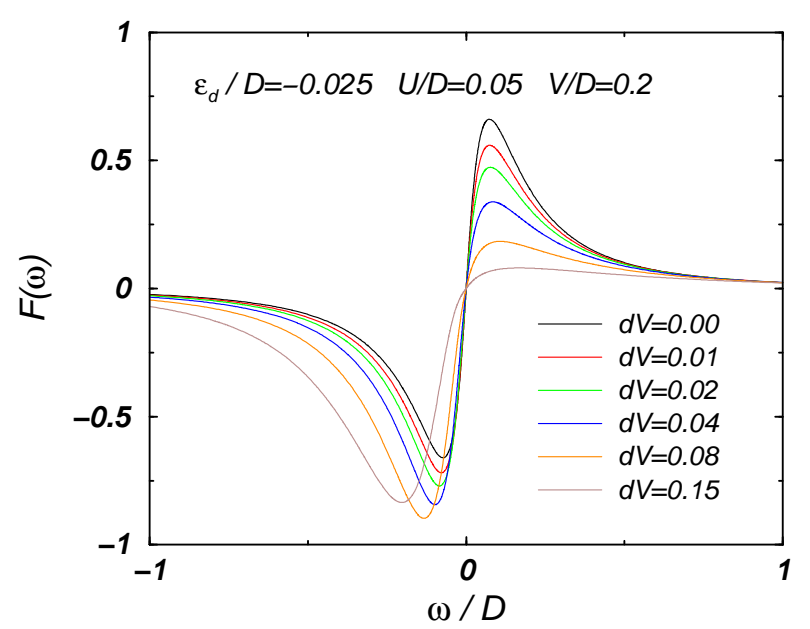

FIG. 5: Local pair correlation, $F(\omega)$ in Eq. (4) as function of $d V$ for $\epsilon=-0.025, U=0.05$ and $V=0.2$.

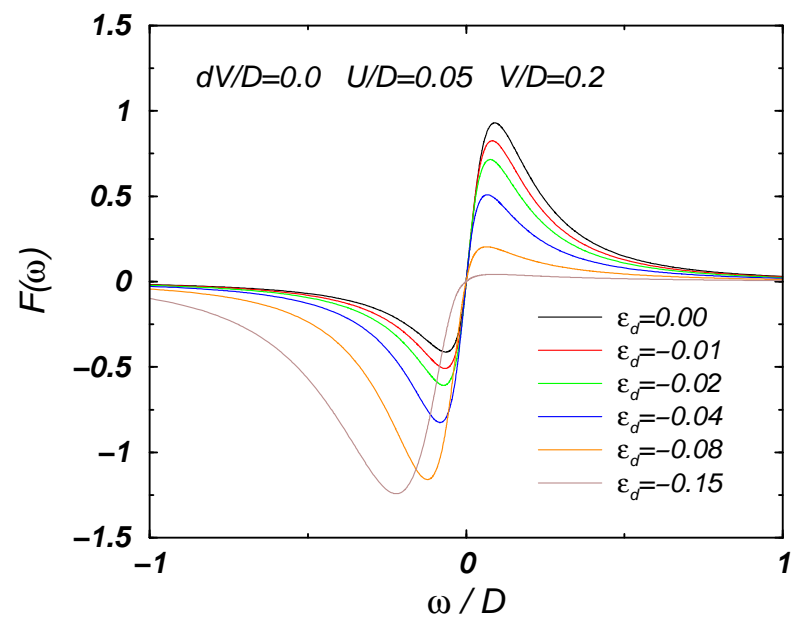

FIG. 6: Local pair correlation, $F(\omega)$ in Eq. (4) as function of $\epsilon$ for $d V=0, U=0.05$ and $V=0.2$.

where $c_{\sigma}^{\dagger}=\sum_{k} c_{k \sigma}^{\dagger}$, representing the metal orbital closest to the impurity. The function $F(\omega)$ in Eq. (4) gives the magnitude of the on-site pairing, as present, for instance, in the negative $U$ Anderson's impurity model. The function $F^{\prime}(\omega)$ describes off diagonal pairing. Virtual Cooper pairs resonate between the on-site position and that in which one component of the pair is at the impurity and the other is in the metal.

We first analyze the function $F(\omega)$, which is a measure of the on-site pairing. The function $F(\omega)$ is shown in Fig. [5 as function of the value of $d V$. For comparison, we plot the same function for $d V=0$ for different values of $\epsilon$ in Fig. 6]. The two functions show similar

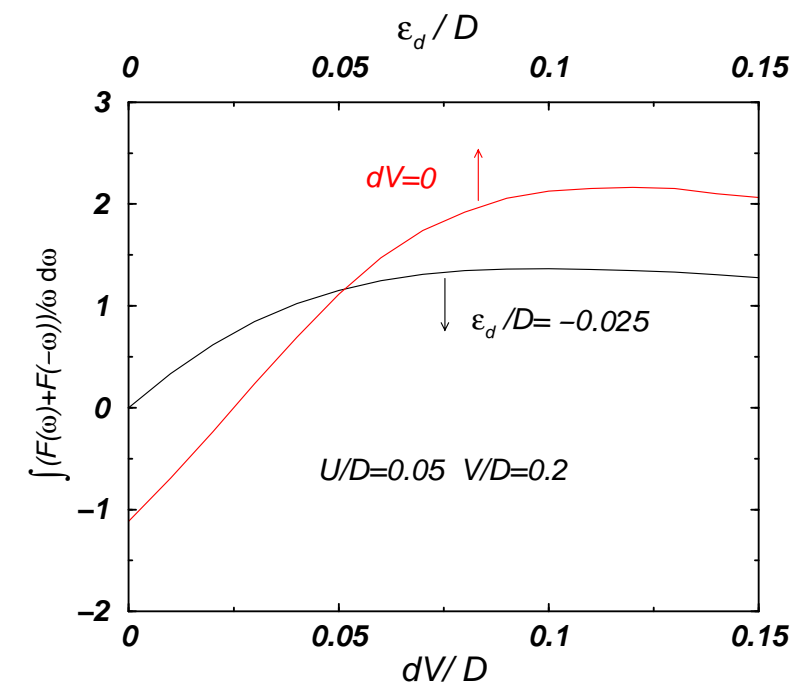

FIG. 7: On site pair susceptibility, $\mathcal{F}$, as defined in Eq. (5) as function of $\epsilon$ for $d V=0$ (top), and as function of $d V$ for $\epsilon=-0.025$ (bottom).

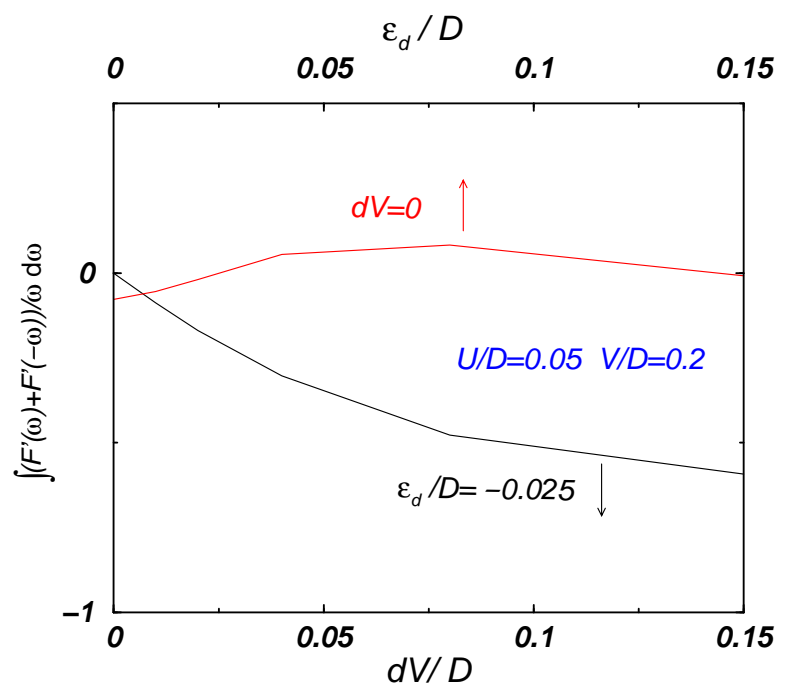

FIG. 8: Off diagonal pair susceptibility, $\mathcal{F}^{\prime}$, as defined in Eq. (5) as function of $\epsilon$ for $d V=0$ (top), and as function of $d V$ for $\epsilon=-0.025$ (bottom).

behavior, suggesting that the main effect of the assisted hopping term on the on-site pairing correlations is the renormalization of the level, since its effect is difficult to be distinguished from the effect of a change in the impurity electron level.

We estimate the tendency towards pairing by using 
Eqs.(4) to define generalized susceptibilities:

$$
\begin{aligned}
\mathcal{F} & =\int_{0}^{\infty} d \omega \frac{F(\omega)+F(-\omega)}{\omega} \\
\mathcal{F}^{\prime} & =\int_{0}^{\infty} d \omega \frac{F^{\prime}(\omega)+F^{\prime}(-\omega)}{\omega}
\end{aligned}
$$

These functions measure the tendency towards the different types of pairing. Fig. [7] gives $\mathcal{F}$ as function of $\epsilon$ for $d V=0$, and as function of $d V$ for $\epsilon=-0.025$. As it can be appreciated, the inclusion of the assisted hopping term $d V$ does not change significantly the value of this susceptibility. Moreover, its magnitude is not too different from the value obtained for $d V=0$. On the other hand, Fig. 8 gives $\mathcal{F}^{\prime}$ as function of $\epsilon$ for $d V=0$, and as function of $d V$ for $\epsilon=-0.025$. In this case, the effects of $d V$ are quite significant. While of $d V=0$ this susceptibility remains close to zero for the whole range of values os $\epsilon$ studied, it raises swiftly as soon as $d V$ is finite. Hence, the assisted hopping term induces an off diagonal pairing term of the type described by $\mathcal{F}^{\prime}$ given in Eq.(4).

This is our main result. In contrast to the local pairing on the $d$-level -which can be understood as the consequence of the primary effect, the renormalization of the level- the off diagonal pairing correlations are increased by the enhanced hopping rate which allows stronger fluctuations if the spin of the $d$ electron and the local conduction electron have opposite orientations. There is another interesting feature of this result: Though the formation of the local moment is strongly suppressed and the impurity has no well defined spin state, the off diagonal correlations are still enhanced. This would indicate that this type of pairing manifests itself in a dynamical way: The spin state of both the local level and the conduction electron state fluctuate, but in a correlated way.

\section{CONCLUSIONS.}

We have studied, using the Numerical Renormalization Group method, the Anderson impurity model with a hopping which depends on the charge state of the impurity. This is the simplest model which includes information about the internal structure of the impurity, beyond a single, rigid, electronic state.

Our results indicate that, when the assisted hopping is sufficiently strong, the model shows a crossover to a phase with off diagonal pairing correlations. It would be interesting to know if this regime could be realized in mesoscopic devices, where the internal structure of a quantum dot can be important.

\section{ACKNOWLEDGEMENTS.}

We are thankful to J. E. Hirsch and to T. Stauber for a critical reading of the manuscript and for helpful comments. Financial support from MCyT (Spain), through grant no. MAT2002-04095-C02-01 is gratefully acknowledged. L.B. acknowledges the financial support provided through the European Community's Research Training Networks Programme under contract HPRN-CT-200200302, Spintronics and Hungarian Grants (OTKA) No. T046303, T034243 and T038162.
1 P. W. Anderson, Phys. Rev. 124, 41 (1961).

2 J. Kondo, Prog. Theor. Phys. 32, 37 (1964).

3 A. C. Hewson, The Kondo problem to Heavy Fermions (Cambridge U. P., Cambridge (UK), 1997).

${ }^{4}$ D. Goldhaber-Gordon, H. Shtrikman, D. Mahalu, D. Abusch-Magder, U. Meirav, and M. A. Kastner, Nature 391, 156 (1998).

5 S. M. Cronenwett, T. H. Oosterkamp, and L. P. Kouwenhoven, Science 281, 540 (1998).

6 D. V. Averin and K. K. Likharev, in Mesoscopic Phenomena in Solids, edited by B. L. Altshuler, P. A. Lee, and R. A. Webb (Elsevier, Amsterdam, 1991).

7 J. Hubbard, Proc. Roy. Soc. (London) 276, 238 (1963).

8 J. Kanamori, Prog. Theor. Phys. 30, 275 (1963).

9 J. E. Hirsch and F. Marsiglio, Phys. Rev. B 43, 424 (1991).

10 J. E. Hirsch, Phys. Rev. B 48, 3327 (1993).

11 M. Ueda and F. Guinea, Zeits. für Phys. B 85, 413 (1991).

12 E. Bascones, C. P. Herrero, F. Guinea, and G. Schön, Phys. Rev. B 61, 16778 (2000).

13 P. Nozières and C. T. deDominicis, Physical Review 178,
1097 (1969).

14 F. Guinea, Phys. Rev. B 67, 195104 (2003).

15 T. Stauber and F. Guinea, Physical Review B 69, 035301 (2004).

16 F. Wegner, Ann. Phys. 3, 77 (1994).

17 F. Wegner, Physics Reports 348, 77 (2001).

18 S. K. Kehrein and A. Mielke, J. Phys. A: Math. Gen. 27, 4259 (1994).

19 S. K. Kehrein and A. Mielke, Ann. Phys. (NY) 252, 1 (1996).

20 P. Lenz and F. Wegner, Nucl. Phys. B 482 [FS], 693 (1996).

21 A. Mielke, Ann. Phys. (Leipzig) 6, 215 (1997).

22 S. K. Kehrein and A. Mielke, Ann. Phys. (Leipzig) 6, 90 (1997).

23 K. G. Wilson, Rev. Mod. Phys. 47, 773 (1975).

24 T. A. Costi, in Density Matrix Renormalization, edited by I. P. et al. (Springer, 1999). 